\title{
Uso de doadores falecidos com anti-HBc positivo no transplante hepático
}

\author{
Use of deceased donors with anti-HBc-positive in liver transplantation \\ Uso de donantes fallecidos con anti-HBc positivo en el trasplante hepático
}

Recebido: 31/05/2021 | Revisado: 09/06/2021 | Aceito: 14/06/2021 | Publicado: 28/06/2021

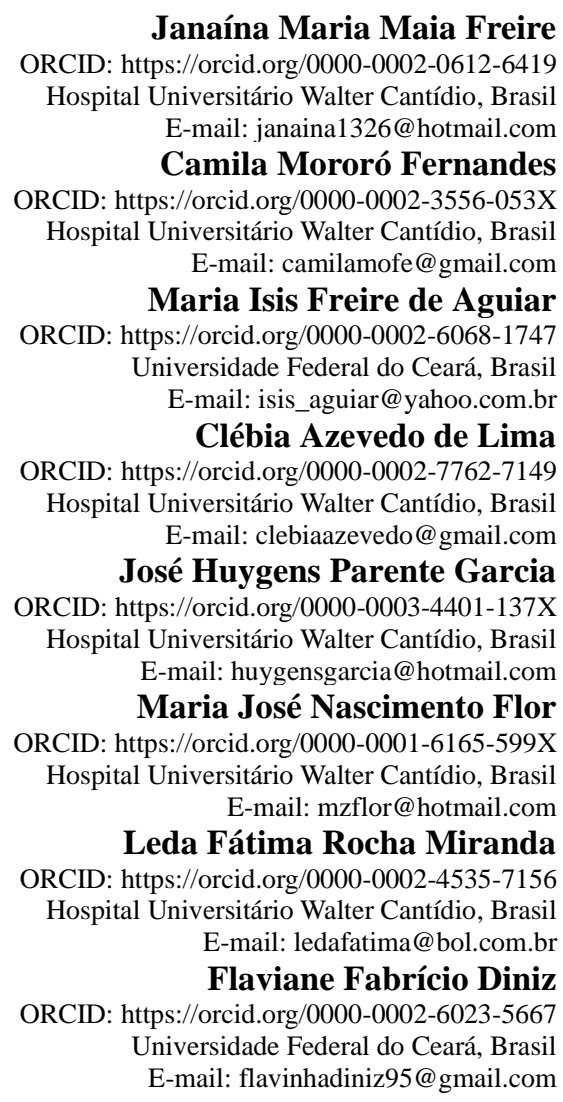

\section{Resumo}

Objetivo: Analisar os resultados do uso de enxertos de doadores com o marcador sorológico anti-HBc positivo em receptores de transplante de fígado. Metodologia: Estudo retrospectivo com 64 receptores de transplante hepático que receberam enxertos de doadores anti-HBc positivo, entre o período de janeiro de 2002 a dezembro de 2017, em um hospital universitário de Fortaleza-CE. A coleta de dados foi realizada com instrumento próprio a partir de prontuários, incluindo dados demográficos e clínicos, como o uso de profilaxia antiviral e o desenvolvimento de hepatite B no pós-transplante. Os dados foram processados no SPSS com análise estatística. Resultados: O sexo masculino foi predominante, a idade média foi de 49,2 anos e as principais indicações para o transplante foram as hepatites virais B e C. Entre os pacientes que eram HBsAg reagentes (25/64) no pré-transplante, apenas um apresentou carga viral detectável no pós-transplante. Entre os receptores HBsAg não reagentes (39/64), foram observados nove $(23,07 \%)$ casos de hepatite B de novo, sendo que cinco deles possuíam anti-Hbs reagente no prétransplante. Todos os noves pacientes apresentaram elevação das transaminases hepáticas e dois evoluíram para óbito. Considerações finais: Para maior segurança, algumas estratégias são indicadas como o uso preferencial de enxertos de doador com anti-HBc positivo em receptores já HBsAg positivo, como o uso de um esquema profilático adequado e um seguimento sorológico periódico no pós-transplante.

Palavras-chave: Doadores de órgãos; Hepatite B; Transplante de fígado.

\section{Abstract}

Aim: To analyze the results of the use of grafts from donors with the anti-HBc-positive serological marker in liver transplantation recipients. Methodology: Retrospective study with 64 liver transplantation recipients who received grafts from anti-HBc-positive donors, between January 2002 and December 2017, in a university hospital in Fortaleza, CE. Data collection was performed with a custom-made instrument based on medical records, including 
demographic and clinical data, as the use of antiviral prophylaxis and the development of hepatitis B in the posttransplant period. The data were processed in SPSS with statistical analysis. Results: Males were predominant, the average age was 49.2 years, and the main indications for the transplantation were viral hepatitis B and C. Among the patients who were HBsAg-reactive (25/64) in the pre-transplant, only one presented detectable viral load in the posttransplant period. Among the HBsAg-nonreactive recipients (39/64), nine (23.07\%) cases of de novo hepatitis B were observed, five of which had anti-HBs-reactive in the pre-transplant. All nine patients presented elevation of hepatic transaminases, and two died. Final considerations: For greater safety, some strategies are indicated, such as the preferential use of grafts from a donor with anti-HBc-positive in HBsAg-positive recipients, as the use of an appropriate prophylactic regimen and periodic serological follow-up in the post-transplant period.

Keywords: Organ donors; Hepatitis B; Liver transplantation.

\section{Resumen}

Objetivo: Analizar los resultados del uso de injertos de donantes con el marcador serológico anti-HBc positivo en receptores de trasplante de hígado. Metodología: Estudio retrospectivo con 64 receptores de trasplante hepático que recibieron injertos de donantes anti-HBc positivo entre enero de 2002 y diciembre de 2017 en un hospital universitario en Fortaleza, CE. La recopilación de datos se realizó con instrumento propio a partir de historiales clínicos, incluyendo datos demográficos y clínicos, como el uso de profilaxis antiviral y el desarrollo de hepatitis $\mathrm{B}$ en el postrasplante. Los datos se procesaron en el SPSS con análisis estadístico. Resultados: El sexo masculino fue predominante, la edad media fue de 49,2 años y las principales indicaciones para el trasplante fueron las hepatitis virales B y C. Entre los pacientes que eran HBsAg reactivos (25/64) en el pretrasplante, solo uno presentó carga viral detectable en el postrasplante. Entre los receptores HBsAg no reactivos (39/64), se observaron nueve $(23,07 \%)$ casos de hepatitis B de novo, de los cuales cinco poseían anti-HBs reactivo en el pretrasplante. Todos los nueve pacientes presentaron elevación de las transaminasas hepáticas y dos murieron. Consideraciones finales: Para una mayor seguridad, se indican algunas estrategias como el uso preferencial de injertos de donante con anti-HBc positivo en receptores ya $\mathrm{HBsAg}$ positivo, como el uso de un régimen profiláctico adecuado y un seguimiento serológico periódico en el postrasplante.

Palabras clave: Donantes de órganos; Hepatitis B; Trasplante de hígado.

\section{Introdução}

Desde a introdução do transplante de fígado como uma modalidade de tratamento, a escassez de órgãos se tornou um grande desafio frente à disparidade entre o número de doadores e o de receptores na lista de espera. Em 2018, no Brasil, havia uma necessidade estimada de 5.192 transplantes hepáticos, porém menos da metade (2.182) foram realizados (ABTO, 2018). Em decorrência disso, muitos centros transplantadores passaram a considerar o uso de órgãos com critérios expandidos, como por exemplo, de doadores idosos, com esteatose ou com algum potencial de transmissão de infecções, dentre elas a hepatite viral (Avelino-Silva et al., 2010).

O DNA do vírus da hepatite B (VHB) pode persistir no soro ou no fígado de indíviduos que são HbsAg (-) mas anti$\mathrm{HBc}(+)$ e enxertos de doadores com este perfil sorológico podem transmitir o vírus. O principal risco relacionado a esses doadores é a possível ativação do VHB, resultando em infecção do órgão transplantado, o que é chamado de hepatite $\mathrm{B} d e$ novo e está associado com hepatite grave e perda do enxerto. $\mathrm{O}$ vírus também pode estar oculto ou latente no receptor e pode ser reativado pela imunossupressão no pós-transplante (Bortoluzzi et al., 2013; Chen et al., 2002).

O risco de desenvolver essa infecção, na ausência da profilaxia, está estritamente relacionado à sorologia prétransplante do receptor. O risco é baixo (0\% e 5\%) entre os pacientes que são transplantados para doença hepática relacionada ao VHB ou com anticorpos anti-HBc e anti-HBs; intermediário (10\% e 18\%) em receptores anti-HBc ou anti-HBs positivos, e alta (70\% e $80 \%)$ em receptores sem qualquer marcador sorológico para o vírus (Bortoluzzi et al., 2013).

A maneira mais simples de prevenir a infecção pelo VHB no pós-transplante é excluir doadores de fígado anti-HBc (+). No entanto, em áreas altamente endêmicas para hepatite B e que sofrem de uma acentuada escassez de doadores, essa exclusão é impraticável. Estudos realizados em regiões diferentes do Brasil mostram que entre 3,2\% a 46,1\% dos indivíduos possuem anti-HBc positivo (Silva et al., 2015; Marca \& Weidlich, 2016; Martins et al., 2015).

Várias outras terapias profiláticas têm sido usadas para prevenir a hepatite B de novo, como por exemplo, a monoprofilaxia com imunoglobulina humana (HBIG), as drogas antivirais isoladas ou em terapia combinada com a HBIG, 
porém ainda não é possível definir qual a estratégia mais segura a ser utilizada em receptores de enxertos anti-HBc positivos (Avelino-Silva et al., 2010; Bortoluzzi et al., 2013).

Portanto, existe uma crescente necessidade de órgãos para transplantes inviabilizando a exclusão do uso de doadores com critérios expandidos, porém no Brasil há poucos estudos sobre o uso de doadores anti-HBc (+) no transplante hepático. Assim, o estudo teve como objetivo analisar os resultados do uso de enxertos de doadores com o marcador sorológico antiHBc positivo em receptores de transplante de fígado.

\section{Metodologia}

Tratou-se de um estudo de natureza quantitativa, retrospectivo e descritivo-analítico, realizado no Hospital Universitário Walter Cantídio (HUWC), instituição vinculada a Universidade Federal do Ceará (UFC) e localizada na cidade de Fortaleza - CE, Brasil.

Na pesquisa quantitativa, através da coleta de dados quantitativos ou numéricos, são gerados conjuntos ou massas de dados que são analisados por técnicas matemáticas como as porcentagens, estatísticas, probabilidades, entre outros. Esse enfoque matemático é importante para possibilitar a previsão de determinados acontecimentos (Pereira et al., 2018).

A população foi composta por todos os receptores de enxertos hepáticos de doadores falecidos com o marcador sorológico anti-HBc (+), transplantados no período de janeiro de 2002 a dezembro de 2017, o que totalizou 75 pacientes.

Foram incluídos na amostra os receptores de ambos os sexos, de qualquer idade que tivessem recebido enxerto de um doador com sorologia positiva para anti-HBc e que tinham acompanhamento regular no pós-transplante. Dez pacientes foram excluídos por óbito com menos de 60 dias após a cirurgia e um paciente por ter realizado transplante duplo fígado-rim. Assim, a amostra do estudo contou com 64 receptores.

A coleta de dados foi realizada nos meses de setembro e outubro de 2019 e utilizou um instrumento de coleta desenvolvido pelos próprios pesquisadores do estudo com variáveis relacionadas ao doador (dados demográficos, causa da morte, tempo de internamento, uso de drogas vasoativas e exame sorológico) e ao receptor (dados demográficos, histórico da doença hepática, perfil sorológico e comorbidades).

Também foi analisado o uso de profilaxia antiviral nos receptores, a ocorrência de hepatite B no pós-transplante, tratamento e resultado da biopsia hepática. A definição de hepatite B de novo utilizada no presente estudo foi a detecção do antígeno da superfície da hepatite B (HBsAg) e/ou carga viral sérica do vírus detectável após o transplante de fígado em receptores previamente HBsAg negativos (Xi et al., 2013).

A análise dos dados foi realizada com suporte do programa Statistical Package for the Social Sciences (SPSS), versão Statistics 22. Na análise estatística descritiva, as variáveis categóricas foram representadas por meio de frequência absoluta (n) e relativa (\%), média e desvio-padrão (DP). A análise inferencial foi realizada através do Teste Exato de Fisher.

O estudo respeitou os requisitos da resolução CNS 466/12 e suas complementares que regulamentam a pesquisa em seres humanos. Foi submetido e aprovado pelo Comitê de Ética do Hospital Universitário Walter Cantídio, com o parecer $n^{\circ}$ 3.516 .362 .

\section{Resultados}

Entre janeiro de 2002 a dezembro de 2017 foram realizados 75 transplantes hepáticos utilizando doadores falecidos com anti-HBc (+), o que corresponde a 5,04\% dos 1486 transplantes realizados pela instituição no período. Destes, 64 foram incluídos na amostra do estudo. 
Os doadores eram em sua maioria do sexo masculino (68,8\%), com uma média de idade de 50,6 anos e as principais causas de morte encefálica foram o acidente vascular cerebral $(59,4 \%)$ e o traumatismo cranioencefálico $(32,8 \%)$. O tempo de internação médio foi de 4,5 dias e 89,1\% estavam em uso de drogas vasoativas no momento da captação do órgão.

Entre os receptores dos enxertos hepáticos, houve um predomínio do sexo masculino $(68,7 \%)$ e uma idade média de 49,2 anos. A principal indicação para o transplante foi a cirrose em decorrência das hepatites virais. No total, 26 pacientes apresentavam carcinoma hepatocelular associado a doença hepática crônica (40,6\%). Além da doença hepática, os pacientes ainda tinham algumas comorbidades relacionadas como a hipertensão arterial sistêmica (15,6\%), o diabetes mellitus (12,5\%) e alteração da função renal $(6,2 \%)$ (Tabela 1$)$.

Tabela 1. Caracterização dos receptores de enxertos hepáticos de doadores anti-HBc positivo $(n=64)$, Fortaleza-CE, Brasil, 2021

\begin{tabular}{lll}
\hline Variável & $\boldsymbol{f ( \% )}$ & Média \pm DP* \\
\hline Sexo & $44(68.7 \%)$ & $49.2 \pm 13.1$ \\
Masculino & $20(31.3 \%)$ & \\
Feminino & \\
Idade (anos) & \\
Doença hepática & $16(25 \%)$ \\
Cirrose por vírus C & $14(21.9 \%)$ \\
Cirrose por vírus B + D & $9(14 \%)$ \\
Cirrose alcoólica & $8(12.5 \%)$ \\
Cirrose por vírus B & $5(7.8 \%)$ \\
Cirrose criptogênica & $5(7.8 \%)$ \\
Cirrose vírus C + álcool & $3(4.7 \%)$ \\
Hepatite autoimune & $3(4.7 \%)$ \\
Cirrose vírus B + álcool & $1(1.6 \%)$ \\
Síndrome Budd-Chiari & $26(40.6 \%)$ \\
Carcinoma Hepatocelular & \\
Comorbidades & $10(15.6 \%)$ \\
Hipertensão arterial sistêmica & $8(12.5 \%)$ \\
Diabetes mellitus & $4(6.2 \%)$ \\
Alteração de função renal &
\end{tabular}

*DP - desvio padrão. Fonte: Dados da pesquisa.

Dos 25 pacientes portadores de hepatite B no pré-transplante (HBsAg reagente), a maioria já realizava tratamento antiviral (84\%) e $40 \%$ tinham carga viral não detectável na fase pré-transplante. Os antivirais mais utilizados foram o entecavir (13/21) seguido pela lamivudina (06/21) e pelo tenofovir (02/21). A profilaxia para recidiva do vírus B no pós-transplante foi a terapia combinada com imunoglobulina humana contra hepatite B (HBIG) e análogos de nucleotídeos. No pós-transplante, um paciente teve registro de carga viral detectável mesmo em uso da profilaxia. O mesmo tinha carga viral indetectável no momento do transplante e realizava a profilaxia combinada com HBIG e lamivudina (150 mg/dia).

Dos 64 receptores, 39 (60,9\%) deles tinham como indicação para o transplante causa não relacionada ao vírus da hepatite B, ou seja, eram HBsAg (-) no pré-transplante. Entre esses indivíduos, 17 (43,6\%) eram anti-HBs reagente 
isoladamente, $12(30,8 \%)$ tinha a combinação de anti-HBs e anti-HBc reagentes, $05(12,8 \%)$ eram apenas anti-HBc reagentes e $04(10,25 \%)$ negativos para ambos os marcadores sorológicos. Em um dos indivíduos não foi informado com relação ao antiHBs e anti-HBc.

Entre os indivíduos que não possuíam hepatite B, houve nove casos de pacientes que converteram o HBsAg para positivo no pós-transplante. As principais diferenças entre os dois grupos são descritas na Tabela 2. Nos pacientes que desenvolveram hepatite B de novo em comparação com os que não desenvolveram, foi observada uma menor porcentagem de anti-HBs reagente $(55,6 \%$ vs $80 \%)$ e anti-HBc reagente $(22,2 \%$ vs $50 \%)$ no pré-transplante, além de um maior uso de transfusão sanguínea no intra e pós-operatório $(66,7 \%$ vs 40\%). Com relação ao uso da profilaxia, no grupo dos pacientes que desenvolveram hepatite B de novo, oito não realizaram e um fez uso do entecavir, porém com a adesão irregular devido às dificuldades para obter o medicamento. Um dos pacientes também relatou contato sexual de risco.

Tabela 2. Indivíduos transplantados com HBsAg (-) no pré-transplante ( $\mathrm{n}=39$ ), Fortaleza, CE, Brasil, 2021.

\begin{tabular}{|c|c|c|c|}
\hline Variável & $\begin{array}{l}\text { Hepatite B de novo } \\
\text { (n=9) } f(\%)\end{array}$ & $\begin{array}{c}\text { Sem hepatite B de novo } \\
(\mathrm{n}=30) f(\%)\end{array}$ & p-valor* \\
\hline \multicolumn{4}{|c|}{ Anti-HBs pré-transplante } \\
\hline Reagente & $5(55,6 \%)$ & $24(80 \%)$ & 0,197 \\
\hline Não reagente & $3(33,3 \%)$ & $6(20 \%)$ & \\
\hline Não informado & $1(11,1 \%)$ & $0(0.0 \%)$ & \\
\hline \multicolumn{4}{|c|}{ Anti-HBc pré-transplante } \\
\hline Reagente & $2(22.2 \%)$ & $15(50.0 \%)$ & 0,2512 \\
\hline Não reagente & $6(66.7 \%)$ & $15(50.0 \%)$ & \\
\hline Não informado & $1(11.1 \%)$ & $0(0.0 \%)$ & \\
\hline \multicolumn{4}{|l|}{ Transfusão } \\
\hline Sim & $6(66.7 \%)$ & $12(40 \%)$ & 0,2552 \\
\hline Não & $2(22.2 \%)$ & $16(53.3 \%)$ & \\
\hline Não informado & $1(11.1 \%)$ & $2(6,7 \%)$ & \\
\hline \multicolumn{4}{|c|}{ Uso de profilaxia regular } \\
\hline Não & $9(100 \%)$ & $20(66,7 \%)$ & 0,0793 \\
\hline Entecavir & $0(0,0 \%)$ & $1(3,3 \%)$ & \\
\hline Lamivudina & $0(0,0 \%)$ & $9(30 \%)$ & \\
\hline
\end{tabular}

*Teste Exato de Fisher. Fonte: Dados da pesquisa.

O tempo médio para o diagnóstico da hepatite B de novo variou entre 12 a 88 meses (média 39,4) após o transplante, sendo o indivíduo com o período mais longo o que utilizou a profilaxia mesmo irregular. A carga viral para o VHB foi detectável na maioria dos pacientes $(88,8 \%)$, e o $\mathrm{HBeAg}$ foi reagente em $55,5 \%$. O antiviral mais utilizado para o tratamento foi o entecavir. Todos os pacientes apresentaram elevação das transaminases glutâmico-oxalacética (TGO) e glutâmicopirúvica (TGP) antes do diagnóstico de hepatite B de novo. A principal alteração encontrada nas biópsias dos enxertos hepáticos foi a hepatopatia crônica. Dois pacientes evoluíram ao óbito com 30 e 34 meses após o transplante (Tabela 3). 
Tabela 3. Seguimento pós-transplante dos indivíduos com hepatite B de novo $(\mathrm{n}=09)$, Fortaleza, CE, Brasil, 2021

\begin{tabular}{cccccc}
\hline Caso & $\begin{array}{c}\text { Detecção } \\
(\text { meses })\end{array}$ & Carga viral & HBeAg & Tratamento & $\begin{array}{c}\text { Biópsia } \\
\text { hepática }\end{array}$ \\
\hline 1 & 12 & Detectado & Não informado & Entecavir & Hepatopatia crônica \\
2 & 22 & Detectado & Reagente & Entecavir & Não realizada \\
3 & 39 & Detectado & Reagente & Tenofovir & Não realizada \\
4 & 26 & Detectado & Reagente & Entecavir & Hepatopatia crônica \\
5 & 75 & Detectado & Reagente & Entecavir & Esteatose macrovesicular \\
6 & 28 & Detectado & Reagente & Entecavir & Atividade inflamatória leve \\
7 & 88 & Detectado & Não informado & Lamivudina & Hepatopatia crônica \\
8 & 26 & Detectado & Não informado & Lamivudina & Rejeição celular aguda \\
9 & 39 & Não detectado & Não reagente & Lamivudina & Hepatopatia crônica \\
\hline
\end{tabular}

Fonte: Dados da pesquisa.

\section{Discussão}

A prevalência de doadores de órgãos com o marcador sorológico anti-HBc (+) equivale a da população em geral, portanto depende da própria prevalência local da infecção pelo vírus da hepatite B (Bárcena, 2014; Cholongitas, Papatheodoridis \& Burroughs, 2010). Em áreas de baixa endemicidade é cerca 5\%, porém pode chegar a $50 \%$ em regiões endêmicas (Bortoluzzi et al., 2013). No presente estudo, os indivíduos com esse anticorpo corresponderam a 5,04\% de todos os doadores utilizados pelo serviço e esse número reduzido reflete a baixa ocorrência da infecção na região. Em 2018, o Nordeste concentrou apenas 9,9\% dos casos notificados de infecção pelo VHB e a cidade de Fortaleza - CE obteve entre todas as capitais brasileiras a segunda menor taxa de casos confirmados (2,7 casos para cada 100 mil habitantes) (Brasil, 2019).

A caracterização dos doadores anti-HBc(+) descritos no estudo é semelhante ao observados por outros autores de diferentes regiões brasileiras com doadores de órgãos em geral. Destaca-se a predominância do sexo masculino (56,3 a 67,2\%) e o acidente vascular cerebral como principal causa de morte encefálica (35,9 a 56,2\%) (Kock, Santana, Silva, Andrade, \& Santos, 2019; Bertasi et al., 2019; Souza, Lira \& Mola, 2015). Estudos apontam que a prevalência do anti-HBc (+) é geralmente maior entre os indivíduos mais velhos, o que pode explicar a média de idade de 50,6 anos encontrada nessa pesquisa (Scuderi et al., 2011).

No Brasil, as principais causas de indicação ao transplante hepático são as hepatites virais e o uso abusivo do álcool (Aguiar et al., 2018; Mendes, Lopes, Fabbris, Castro-e-Silva Júnior, \& Galvão, 2016; Freitas et al., 2010) similar ao encontrado. Na amostra do estudo, 26 dos receptores $(40,6 \%)$ tinham carcinoma hepatocelular, dados semelhantes são mostrados em outros trabalhos na variação de 38 a 50\% (Bortoluzzi et al., 2013; Meirelles Junior et al., 2015; Souza, Rodriguez, Motta, Brandão, \& Marroni, 2019).

$\mathrm{O}$ vírus da hepatite $\mathrm{B}$ (VHB) é uma das causas mais comuns de doenças crônicas do fígado, incluindo cirrose hepática, insuficiência hepática e carcinoma hepatocelular (CHC). O risco de adquirir uma nova infecção pelo VHB foi reduzido com a introdução do antígeno de superfície da hepatite B (HBsAg) através da vacinação. No entanto, com o advento da infecção oculta pelo VHB, está claro que muitos indivíduos infectados podem não expressar HBsAg, mas podem conter o DNA do VHB (Jahan et al., 2016). 
No centro transplantador do presente estudo, os enxertos hepáticos provenientes de doadores anti-HBc (+) são aceitos para receptores que são portadores de hepatite B ou para aqueles que possuem o anti-HBs reagente, exceto em casos de urgência para o transplante.

Os enxertos hepáticos provenientes de doadores anti-HBc positivo podem ser ofertados em primeiro lugar a receptores com doença hepática relacionada ao VHB, já que eles irão necessitar da terapia antiviral contínua recebendo ou não órgãos com esse perfil (Cholongitas, Papatheodoridis, \& Burroughs, 2010). Nesse estudo, os pacientes realizaram a profilaxia contra a recorrência viral com a imunoglobulina humana para a hepatite B (HBIG) combinada com um análogo de nucleotídeos e apenas um paciente apresentou carga viral detectável após o transplante utilizando a HBIG e lamivudina. Esse esquema combinado parece ser a estratégia efetiva para prevenir recorrência do VHB no pós-transplante (Paraná et al., 2015). Outro estudo relatou casos de recorrência do vírus em indivíduos que utilizaram tanto HBIG ou lamivudina isoladamente como a profilaxia combinada com ambos (Cholongitas, Papatheodoridis, \& Burroughs, 2010).

$\mathrm{O}$ risco de hepatite de novo pós-transplante é a maior limitação do uso de enxertos hepáticos de doadores anti-HBc positivo, já que a infecção viral oculta no doador pode ser reativada devido a terapia imunossupressora (Cholongitas, Papatheodoridis, \& Burroughs, 2010). Estudos internacionais mostram a ocorrência dessa infecção entre 5 a 52,4\% nos receptores previamente HBsAg não reagentes (Xi et al., 2013; Cholongitas, Papatheodoridis, \& Burroughs, 2010; Prieto et al., 2011). No nosso grupo de 39 indivíduos transplantados por doenças não relacionadas ao VHB, ocorreu a soroconversão para HBsAg reagente em nove casos $(23,07 \%)$. Existem algumas vias possíveis para infecção, porém a mais importante é através do doador HBsAg (-) / anti-HBc (+) (Dong et al., 2017).

Entre os receptores com hepatite B de novo, 55,6\% deles eram anti-HBs reagentes. Vários autores apontam que a presença do anticorpo reduz a probabilidade de desenvolver a infecção, mas não a elimina. Há relatos de pacientes que mesmo com esse perfil sorológico apresentam a hepatite B de novo sendo sugerido o uso de profilaxia também nessa população (Chen et al., 2002; Cholongitas, Papatheodoridis, \& Burroughs, 2010). Além disso, é provável que em pessoas imunossuprimidas seja ideal manter títulos maiores desse anticorpo em comparação ao recomendado à população em geral (Bortoluzzi et al., 2013).

Apesar do grupo de pacientes que desenvolveram hepatite $\mathrm{B}$ de novo terem utilizado mais hemocomponentes do que o grupo que não desenvolveu, outro estudo apontou que a quantidade de transfusão sanguínea realizada durante o intraoperatório do transplante não se correlacionou com a ocorrência da infecção (Xi et al., 2013).

As revisões de literatura realizadas com doadores anti-HBc (+) mostraram que o uso de qualquer um dos esquemas profiláticos diminui o risco de desenvolvimento da infecção (Bárcena, 2014). Uma revisão que totalizou 788 receptores observou que a profilaxia pós-transplante afetou significante a probabilidade de desenvolver a hepatite B de novo, sendo a ocorrência em 28,2\% nos que não fizeram e em 8,2\% nos receptores que realizaram ( $<$ < 0.001 ) (Cholongitas, Papatheodoridis, \& Burroughs, 2010).

O tempo médio para a detecção do HBsAg (+) no pós-transplante foi de 39,4 meses (12-88), esse valor foi acima do observado em outros estudos realizados na Europa e Ásia que mostraram médias entre 11,4 a 27 meses e variações de 3,5 a 60 meses (Xi et al., 2013; Prieto et al., 2001; Donataccio et al., 2006). Ainda que os dados sejam escassos e o seguimento dos pacientes sejam variáveis, o aparecimento da hepatite B de novo pode ser tardio como até seis ou sete anos depois do transplante (Bárcena, 2014). A hepatite B de novo desenvolve-se com mais rapidez em pacientes que não fazem a profilaxia em comparação a aquelas que realizam (Cholongitas, Papatheodoridis, \& Burroughs, 2010). No presente estudo, o paciente com o período mais longo para o diagnóstico ( 88 meses) foi o único que utilizou a profilaxia mesmo com má adesão. Devido ao seu custo-benefício, a monoterapia com lamivudina ainda é bastante utilizada como profilaxia, sua duração ainda é incerta, porém a manutenção por tempo indefinido juntamente com o rastreamento sorológico periódico ainda parece ser a melhor estratégia (Avelino-Silva et al., 2010; Bárcena, 2014). 
Se a hepatite B de novo for diagnosticada, o tratamento com antiviral é mandatório. O análogo de nucleotídeo mais utilizado nesse trabalho foi o entecavir. Devido ao perfil de resistência da monoterapia com lamivudina a longo prazo, o entecavir e o tenofovir podem ser os agentes de escolha atual para o tratamento (Cholongitas, Papatheodoridis, \& Burroughs, 2010). Os dados sobre a sobrevida de receptores de enxertos anti-HBc (+) ainda são controversos. Enquanto é apontado que há associação significativa entre o uso desses doadores com piores taxas de sobrevida do receptor e do enxerto no pós-transplante (Lai et al., 2011), em diretrizes nacionais é relatado que com o uso da profilaxia a sobrevida é similar aos 12 meses (86\% em ambos os grupos) e aos 60 meses (68\% anti-HBc positivo; 67\% anti-HBc negativo) (ABTO, 2010).

Embora o estudo tenha considerado a população total de receptores transplantados por um período de 16 anos, considera-se como limitação a reduzida amostra de doadores anti-HBc (+) em virtude da baixa prevalência da infecção pelo vírus da hepatite B no local onde o levantamento foi realizado.

\section{Considerações Finais}

O uso de enxertos hepáticos de doadores anti-HBc (+) se mostrou como uma estratégia para beneficiar indivíduos que necessitam de um transplante e assim diminuir a mortalidade na lista de espera. Para a maior segurança dos receptores algumas estratégias são indicadas como o uso preferencial em indivíduos já HBsAg (+), o uso de um esquema profilático adequado e um seguimento sorológico periódico no pós-transplante. Sugere-se a realização de estudos multicêntricos quanto ao uso desses doadores, incluindo amostra de diferentes estados brasileiros e em regiões onde a prevalência da infecção pela hepatite B seja elevada.

\section{Agradecimentos}

Ao Ministério da Educação pelo financiamento da realização estudo através de bolsa de residência multiprofissional em saúde.

\section{Referências}

Aguiar, M. I. F., Alves, N. P., Braga, V. A. B., Souza, Â. M. A., Araújo, M. Â. M., \& de Almeida, P. C. (2018). Psychosocial aspects of quality of life of hepatic transplant receivers. Texto e Contexto Enfermagem, 27(2), 1-11.

Associação Brasileira de Transplante de Órgãos - ABTO (2010). Doadores limítrofes no transplante de fígado. Revista Da Associação Médica Brasileira, $56(6), 625-630$.

Associação Brasileira de Transplantes de Órgãos - ABTO (2018). Dimensionamento dos transplantes no Brasil e em cada estado (2011 - 2018). Registro Brasileiro de Transplantes. ABTO.

Avelino da Silva, V. I., D’Albuquerque, L. A. C., Bonazzi, P. R., Song, A. T. W., Miraglia, J. L., De Brito Neves, A., \& Abdala, E. (2010). Liver transplant from Anti-HBc-positive, HBsAg-negative donor into HBsAg-negative recipient: Is it safe? A systematic review of the literature. Clinical Transplantation, 24(6), 735-746.

Bárcena, R. (2014). Transmisión de la infección B por injertos anti-HBc positivos. Gastroenterologia y Hepatologia, 37(S2), 43-50.

Bertasi, R. A. O., Bertasi, T. G. O., Reigada, C. P. H., Ricetto, E., Bonfim, K. de O., Santos, L. A., Athayde, M. V. O., Pedrosa, R. B. S., Perales, S. R., Sardinha, L. A. C., Ataide, E. C., Boin, I. F. S. F., \& Hirano, E. S. (2019). Perfil dos potenciais doadores de órgãos e fatores relacionados à doação e a não doação de órgãos de uma Organização de Procura de Órgãos. Revista Do Colégio Brasileiro de Cirurgiões, 46(3), 1-8.

Bortoluzzi, I., Gambato, M., Albertoni, L., Mescoli, C., Pacenti, M., Cusinato, R., Germani, G., Senzolo, M., Rugge, M., Boccagni, P., Zanus, G., Cillo, U., Burra, P., \& Russo, F. P. (2013). Use of grafts from Anti-HBc-positive donors in liver transplantation: A 5-year, single-center experience. Transplantation Proceedings, 45(7), 2707-2710.

Brasil. Ministério da Saúde (2019). Secretária de Vigilância em Saúde. Boletim epidemiológico das hepatites virais. 50(17): 03-71.

Chen, Y. S., Wang, C. C., Villa, V. H., Wang, S. H., Cheng, Y. F., Huang, T. L., Jawan, B., Chiu, K. W., \& Chen, C. L. (2002). Prevention of de novo hepatitis B virus infection in living donor liver transplantation using hepatitis B core antibody positive donors. Clinical Transplantation, 16(6), 405-409.

Cholongitas, E., Papatheodoridis, G. V., \& Burroughs, A. K. (2010). Liver grafts from anti-hepatitis B core positive donors: A systematic review. Journal of Hepatology, 52(2), 272-279. 
Donataccio, D., Roggen, F., Reyck, C., Verbaandert, C., Bodeus, M., \& Lerut, J. (2006). Use of anti-HBc positive allografts in adult liver transplantation: Toward a safer way to expand the donor pool. Transplant International, 19(1), 38-43.

Dong, C., Gao, W., Ma, N., Sun, C., Zheng, W. P., Wang, K., \& Shen, Z. Y. (2017). Risks and treatment strategies for de novo hepatitis B virus infection from anti-HBc-positive donors in pediatric living donor liver transplantation. Pediatric Transplantation, 21(2), 1-6.

Freitas, A. C. T., Itikawa, W. M., Kurogi, A. S., Stadnik, L. G., Parolin, M. B., \& Coelho, J. C. U. (2010). The impact of the model for end-stage liver disease (MELD) on liver transplantation in one center in Brazil. Arquivos de Gastroenterologia, 47(3), 233-237.

Jahan, M., Islam, A., Akbar, S. M. F., Takahashi, K., Tabassum, S., Rahman, A., Haque, A., Biswas, J., Mishiro, S., \& Al-Mahtab, M. (2016). Anti-HBc Screening of Blood Donors in Bangladesh: Relevance to Containment of HBV Propagation. Journal of Clinical and Experimental Hepatology, 6(2), 115-118.

Kock, K. D. S., Santana, M. G., Silva, S. C., Andrade, S. B., \& Santos, E. N. (2019). Perfil epidemiológico, disfunção orgânica e eletrolítica em potenciais doadores de órgãos e tecidos de um hospital do sul do Brasil. Arquivos Médicos Dos Hospitais e Da Faculdade de Ciências Médicas Da Santa Casa de São Paulo, 64(2), 100-107

Lai, Q., Molinaro, A., Spoletini, G., Mennini, G., Grieco, M., Merli, M., Corradini, S. G., Berloco, P.B., \& Rossi, M. (2011). Impact of anti-hepatitis B corepositive donors in liver transplantation: A survival analysis. Transplantation Proceedings, 43(1), 274-276.

Marca, F., \& Weidlich, L. (2016). Soroprevalência em doadores de sangue do Vale do Taquari, RS. Revista Brasileira de Análises Clínicas, 48(3), $240-244$.

Martins, A. P. B., Silva, B., Molin, D. B. D., \& Mendes, G. A. Soroprevalência de doenças infecciosas em doadores de sangue do Hemocentro Regional de Cruz Alta - Rio Grande do Sul. (2015). Clinical \& Biomedical Research, 171(4):211-216.

Meirelles Júnior, R. F., Salvalaggio, P., Rezende, M. B., Evangelista, A. S., Guardia, B. D., Matielo, C. E. L., Neves, D. B., Pandullo, F. L., Felga, G. E. G., Alves, J. A. S., Curvelo, L. A., Diaz, L. G. G., Rusi, M. B., Viveiros, M. M., Almeida, M. D., Pedroso, P. T., Rocco, R. A., \& Meira Filho, S. P. (2015). Liver transplantation: history, outcomes and perspectives. Einstein (São Paulo, Brazil), 13(1), 149-152.

Mendes, K. D. S., Lopes, N. L. C., Fabbris, M. A., Castro-e-Silva Júnior, O., \& Galvão, C. M. (2016). Caracterização sociodemográfica e clínica de candidatos a transplante de fígado. Acta Paulista de Enfermagem, 29(2), 128-135.

Paraná, R., Souto, F., Salcedo, J. M., Dantas, T., Braga, W., Lopes, E., \& Parise, E. R. (2015). Recomendações da Sociedade Brasileira de Hepatologia para diagnóstico e tratamento das hepatites B e Delta.http://sbhepatologia.org.br/pdf/HEPATITE_B_e_DELTA_SBH.pdf.

Pereira, A. S., Shitsuka, D. M., Parreira, F. J., \& Shitsuka, R. (2018). Metodologia da pesquisa científica. [e-book]. Santa Maria. Ed. UAB/NTE/UFSM. https://repositorio.ufsm.br/bitstream/handle/1/15824/Lic_Computacao_Metodologia-Pesquisa-Cientifica.pdf?sequence=1.

Prieto, M., Gómez, M. D., Berenguer, M., Córdoba, J., Rayón, J. M., Pastor, M., García-Herola, A., Nicolás, D., Carrasco, D., Orbis, J. F., Mir, J., \& Berenguer, J. (2001). De novo hepatitis B after liver transplantation from hepatitis B core antibody - positive donors in an area with high prevalence of anti$\mathrm{HBc}$ positivity in the donor population. Liver Transplantation, 7(1), 51-58.

Scuderi, V., Ceriello, A., Santaniello, W., Aragiusto, G., Romano, M., Migliaccio, C., \& Calise, F. (2011). Hepatitis B prophylaxis in hepatitis B-negative recipients transplanted with donor grafts positive for hepatitis B core antibodies. Transplantation Proceedings, 43(1), 271-273.

Silva, A. C. B., Souza, L. F. B., Katsuragawa, T. H., Lima, A. A., Vieira, D. S., \& Salcedo, J. M. V. (2015). Perfil soroepidemiológico da hepatite B em localidades ribeirinhas do rio Madeira, em Porto Velho, Estado de Rondônia, Brasil. Revista Pan-Amazônica de Saúde, 55(69):51-59.

Souza, A. B., Rodriguez, S., Motta, F. L., Brandão, A. B. M., \& Marroni, C. A. (2019). The cost of adult liver transplantation in a referral center in Southern Brazil. Arquivos de Gastroenterologia, 56(2), 165-171.

Souza, B. S. J., Lira, G. G., \& Mola, R. (2015). Notification of brain death in the hospital. Revista Da Rede de Enfermagem Do Nordeste, 16(2), 194-200.

Xi, Z. F., Xia, Q., Zhang, J. J., Chen, X. S., Han, L. Z., Zhu, J. J., Wang, S. Y., \& Qiu, D. K. (2013). De novo hepatitis B virus infection from anti-HBcpositive donors in pediatric living donor liver transplantation. Journal of Digestive Diseases, 14(8), 439-445. 